\title{
Configuration of the posterior lumbar muscles regarding the study of the lumbosacral malformations
}

\author{
D. Recoules-Arche ${ }^{1}$, Omar Husseini ${ }^{2}$, F. Salami ${ }^{2}$, Thierry Somon ${ }^{2}$ \\ 1. Service de chirurgie du rachis, Hôpital J acques Monod, France. 2. Service de radiologie, Hôpital Jacques Monod, France.
}

Correspondence: Thierry Somon. Address: Service de radiologie, Hôpital Jacques Monod, BP 2476083 Le Havre Cedex, France. E-mail: thierrysomon@gmail.com

Received: August 15, 2013

Accepted: December 8, 2013

Online Published: April 10, 2014

DOI : $10.5430 /$ jbgc.v4n2p43

URL: http://dx.doi.org/10.5430/jbgc.v4n2p43

\section{Abstract}

Purpose: A correlation between the shape of the three posterior lumbar muscles (Multifidus, Longissimus pars lumborum and Iliocostalis pars lumborum) and the vertebrae levels has been studied with regards to lumbosacral malformations and the extraforaminal approach (ELIF).

Material and method: During a spine unit consultation, a series of 90 lumbar spines have been studied with X-ray and CT axial horizontal cross sections thanks to a special software. At every level, the ratio between the surface of the Multifidus and the global surface of the 3 posterior lumbar muscles was calculated. Among the 90 studied lumbar spines, 59 presented a normal bony frame while 31 were supposed to have a lumbosacral malformation.

Results: For L5S1, L4L5, L3L4, L2L3 the normal ratio are respectively 95\%, 51\%, 34\% and 18\%. Any changes in these patterns may call forth a sacralization with two different forms, depending on the number (four or five) of the so-called "lumbar vertebrae".

Conclusion: The muscle patterns lead the clinician to study the lumbosacral malformation, and the surgeon to undergo the extraforaminal intermuscular discal surgery.

\section{Key words}

Posterior lumbar muscles, Lumbosacral malformations and the extraforaminal approach, Extraforminal intermuscular discal surgery, Mini invasive technique, Multifidus surface and global surface

\section{Background}

The extraforaminal approach ${ }^{[1,2]}$ to the lumbar spine makes it possible to treat a very lateral herniation or to perform an interbody fusion with cages (ELIF). This approach is made through a natural cleavage plane between the three posterior lumbar muscles (Multifidus [M], Longissimus pars lumborum [L] and Iliocostalis pars lumborum [IC]). The shape of these muscles varies with the level of the lumbar spine. To help the surgeon in this intermuscular approach, at every level we have made a correlation between the shape of these muscles and the level of the vertebral discs. A pattern has been found, at first, in normal lumbar spines, and then also in spines with lumbosacral malformations. 
With lumbosacral malformations, the bony frame is somewhat ambiguous and not always easily described despite a lot of classic markers ${ }^{[3-5]}$. The vertebral segmentation from genetic origin includes both muscular and bony structures. The global study can help the understanding of the malformations.

\section{Anatomy}

Every lumbar vertebra gives insertion to five fascicles of the Multifidus. Every fascicle spans the underlying vertebrae. The belly of the Multifidus lies behind the vertebral lamina as far as the mamillary processes. The last mamillary process is the sacral one, near the ala sacra and the internal iliac aisle. The length of the posterior vertebral lamina increases from L1 to S1. So, the belly of the Multifidus is larger in S1 than in L1.

The Longissimus and Iliocostalis (pars lomborum) have one fascicle at each level. Each fascicle attaches to a transverse process. The muscular belly spans to the iliac crest and the postero-superior iliac spine, so their bellies are largest in the middle lumbar level and decrease towards the iliac crest, where they attach and form a sheet known as the musculoaponeurotic lamina. It must be noted that the inversion of the muscular shapes are larger in the upper level for the Iliocostalis and the Longissimus (pars lumborum), and larger in L5S1 for the Multifidus.

\section{Material and method}

During a spine unit consultation, a series of 90 lumbar spines have been analyzed by several experienced spine specialists: one neurosurgeon and a few neuroradiologists. The results of each specialist have been matched a second time. The study was made with X-ray and CT-scans using a special software.

Among the 90 studied lumbar spines, 59 presented a normal bony frame, while 31 had lumbosacral malformations. A disc above a vertebra that is attached to the pelvis is named "free or functional disc". The first "free disc" is usually L5S1. The upper discs, named 2nd, 3rd and 4th discs are usually L4L5, L3L4 and L2L3 (see Table 1).

Table 1. False or nonfunctional disc and free disc

\begin{tabular}{llll}
\hline & & Normal lumbar spine & Lumbar spine with sacralization \\
\hline False or nonfunctional disc & & S1S2 & L5S1 \\
& 1st disc & L5-S1 & L4-L5 \\
Free disc & 2nd disc & L4-L5 & L3-L4 \\
& 3rd disc & L3-L4 & L2-L3 \\
& 4th disc & L2-L3 & \\
\hline
\end{tabular}

A disc under a vertebra which is completely or partially attached to the pelvis is named "false or non functional disc". Usually, this disc is S1S2. The surface of the posterior muscles has been measured at the level of the four last "free discs", and if necessary, have the "non functional disc" with horizontal cross-sections that go through the posterior part of the inferior endplate. These horizontal cross-sections are studied from native slides acquired during the CT scan exam and not from cross-sections made in the axis of the disc. The axis of the discs varies with the lumbar lordosis. The cross-sections overlap and represent a shifting surface of the muscles. Horizontal cross-sections, on the other hand, are parallel and represent a specific surface of the muscle by definition.

Two muscular surfaces have been measured: one including the surface of the Multifidus (MS) alone, and another including the global surface (GS) of the three muscles (M + L + IC). The ratio of these two surfaces (MS/GS) has been calculated. The vertebrae have been named in accordance with classic data:

Five free lumbar vertebrae:

- The L3 transverse process is longer, larger and more horizontal than any other 
- On the other hand, the L4 transverse process is short and slender with an oblique ascending slope

- The innominate line crosses the disc L5S1 in its posterior part, on the lateral X-Ray

- The sacral slope (S1 endplate) is $38^{\circ}\left( \pm 8^{\circ}\right)$

- The superior attach of the psoas muscle is at the T12L1 level

\section{Results}

The results are reported in Table 2.

Table 2. Surface ratio of the lumbar posterior muscles at every disc level

\begin{tabular}{llll}
\hline & & Normal lumbar spine & Sacralization \\
\hline Number of spines & & 59 & 31 \\
False or nonfunctional disc & & $100 \%$ & $100 \%$ \\
& 1 st disc & $95 \%(80 \%-100 \%)$ & $64 \%(43 \%-81 \%)$ \\
Free disc & 2nd disc & $51 \%(43 \%-66 \%)$ & $37 \%(33 \%-42 \%)$ \\
& 3rd disc & $34 \%(27 \%-39 \%)$ & $20 \%(15 \%-27 \%)$ \\
& 4 th disc & $18 \%(15 \%-21 \%)$ & $15 \%(12 \%-18 \%)$ \\
\hline
\end{tabular}

\section{Discussion}

At every level, the mean ratio between the surface of the Multifidus and the global surface of the three muscles is very precise, with a very narrow range. To be the most exact, the extreme values, at each level, never overlap the values of the adjacent levels. The smallest range is at the L3L4 level. This can be explained by the lumbar lordosis. The L3L4 disc is usually in a horizontal plan. A horizontal cross-section is not involved by the lordosis. The sections are nearly perpendicular to the muscles, and as we wrote before, they always have the same angle. At every level, a precise pattern can be described as the pattern of a specific segment. If, however, the figures do not match the presumed level, a malformation can be suspected. The true level can be found in Table 1.

Other authors have studied the posterior lumbar muscles. Fortin $\mathrm{M}$ et al. ${ }^{[7]}$ have described a quantitative paraspinal measurement. Hoh et al. ${ }^{[8]}$ have searched the anatomical features of the paramedian muscles with application to the splitting approach to the lumbar spine. If we compare the results we have obtained with this new method to the classic anatomical markers, we notice that all classic markers can be wrong but one; the superior attachment of the psoas muscle.

To conclude, it is the muscular system or myomer, with its psoas muscle forward and the posterior lumbar muscles backward, that marks the segmentation most precisely. The disc, L3L4, or the 3rd "free disc" is the most reliable (MS/GS $34 \%)$. This disc is the key of our research.

In our study, we find 31 sacralizations for 90 lumbar spines. Two forms of sacralizations can be described. The first one (see Figure 1) is a lumbar spine with 4 free vertebrae and 1 vertebra which is attached to the pelvis. It is an obvious sacralization, clearly displayed by the radiologist X-Ray account. We named this form, 4FV +1 (4 free vertebrae + 1 attached). At every level, the ratio (those of the free discs and those of the transitional disc) are obtained by a normal lumbar spine from L2L3 to L5S1. Thirteen forms of this sacralization $(4 \mathrm{FV}+1)$ have been found on 31 various sacralizations.

The second form of sacralization (see Figure 2) represents the other 18 sacralizations. This form, unlike the sacralization $4 \mathrm{VF}+1$, is far from obvious. At first glance, the lumbar spine presents five free lumbar vertebrae and one transitional 
vertebra. For this reason, we named this form $5 \mathrm{FV}+1$. At every level, the ratios are different from those obtained in a normal lumbar spine. Or, as we have written, the ratios are very precise, specific for each level. The discrepancy has prompted us to search for a hidden malformation.
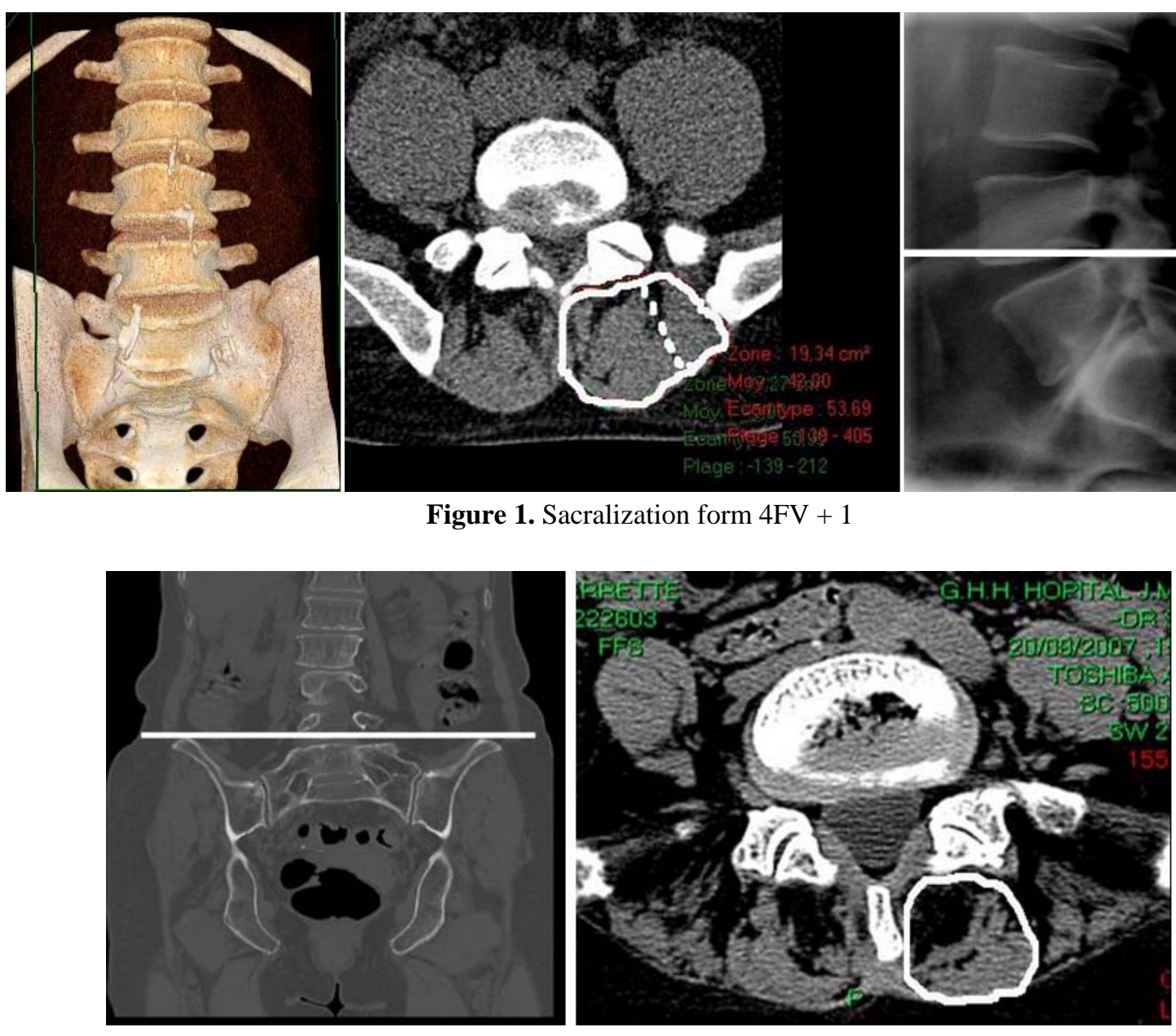

Figure 2. Sacralisation form $5 \mathrm{FV}+1$

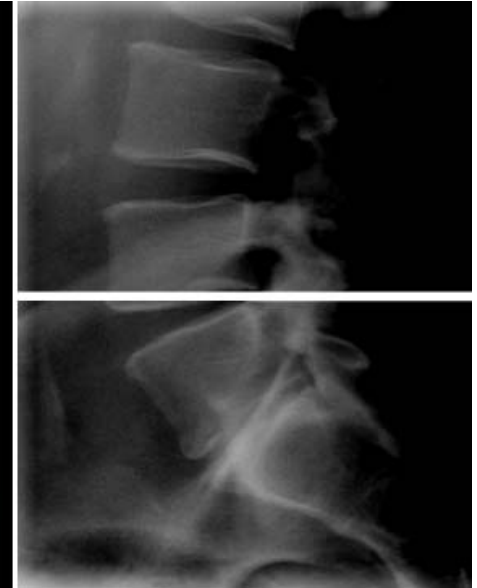

Figure 1. Sacralization form $4 \mathrm{FV}+1$

For these 18 lumbar spines, we studied the insertion of the psoas muscle, either with a MRI or by coronal cross-sections. In case of doubt, the global counts of the thoracic vertebrae have been searched. The findings revealed:

- $\quad$ The first free vertebra, under the rib cage, is not L1 but T12.

- The last free vertebra, at the lumbosacral junction, is not L5 but L4.

This form of sacralization $(5 \mathrm{FV}+1)$ also presents some particularities like the last free vertebra has a transverse process, which is smaller than the next superior vertebra which is the second free vertebra. This last one often presents the longest transverse process (instead, these particularities are usually those of L4 and L3 respectively). The innominate line, on the lateral X-Ray view does not cross the last free disc but does cross the non functional disc (this particularity usually belongs to L4L5 and L5S1). Thus, this form of sacralization 5FV +1 is obviously the most difficult one to identify. The radiologists describe this form as a normal lumbar spine or a kind of lombalization.

According to Hox, genes encode transcription factors which are essential for patterning the anterior to posterior animal body axis ${ }^{[9,10]}$. During development, hox genes are activated in a time sequence. A slight time delay in expression leads to caudal transposition of the sacrum. When we look at primates ${ }^{[11]}$, we see that primitive monkeys (macaque, gibbon) have 
seven or eight free vertebrae (11 thoracic vertebrae, six or seven free lumbar vertebrae and one free sacral vertebra). The lumbar spine mobility between the thorax and the pelvis is important. Great big monkeys (gorilla, chimpanzee), however, stand up, and this required a strong bony pelvic frame for sustaining the weight of the erected trunk. The chimpanzee has 12 or 13 thoracic vertebrae, three free lumbar vertebrae and six or seven sacral vertebrae which are joined. The lumbar spine of homosapiens seems to be placed between these two forms. The lumbar spine flexibility may be linked to the persistence of either one free thoracic vertebrae or one free sacral vertebra (see Figure 3). So the lumbar spine inflexibility may be linked to the complete dorsalization of 12 thoracic vertebrae and the thorax or the progressively upward sacralization, including the 5th or even the 4th lumbar vertebrae.

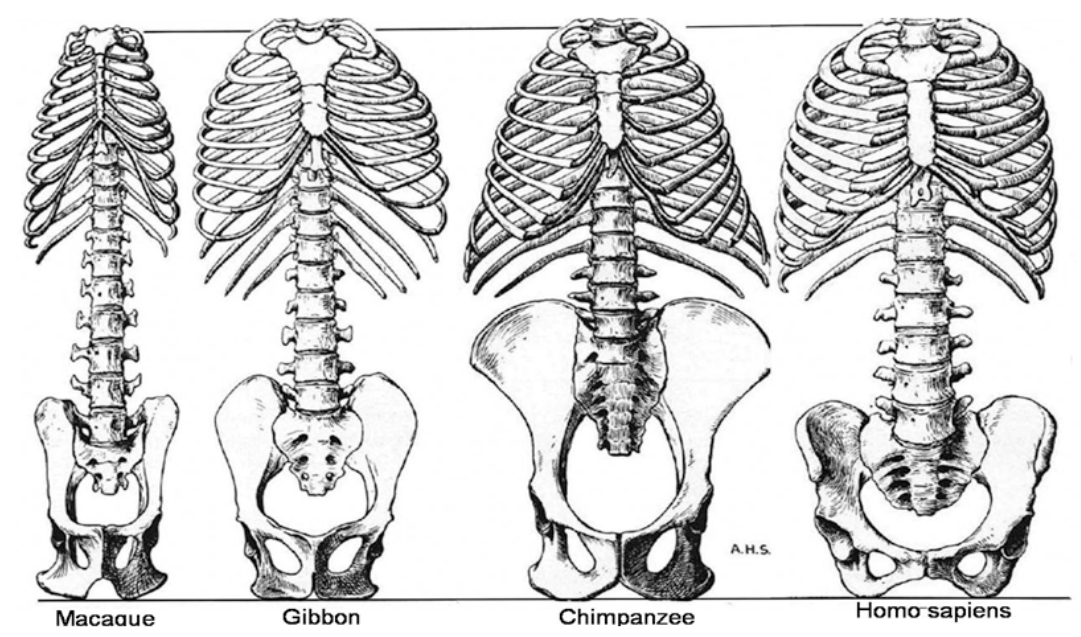

Figure 3. Monkeys and human skeletons

Today, proponents of the lumbar interbody fusion surgery tend to prefer increasingly more lateral approach. The ELIF (see Figure 4) reaches the disc with a $45^{\circ}$ angle, due to a skin incision $10 \mathrm{~cm}$ lateral to the midline. The approach reaches the plane between the Multifidus and the Longissimus pars lumborum ${ }^{[1,2]}$. The surgeon is able to glide between the muscles, described by CT. The surgical approach is led, not by an ambiguous bony frame due to the vertebral distribution abnormalities, but by the pattern of the muscles groups at the level of the foramen. For example, at the L4L5 level, the surgeon will find the plane between the Multifidus and the Longissimus pars lumborum midway between the midline inside and the iliac crest outside. At the L3L4 level, the plane will be at one third of the way from the midline to the iliac crest.

Figure 4. Extraforaminale lumbar interbody fusion

Note. The approach to the foramen through the intermuscular plane with a $45^{\circ}$ angle

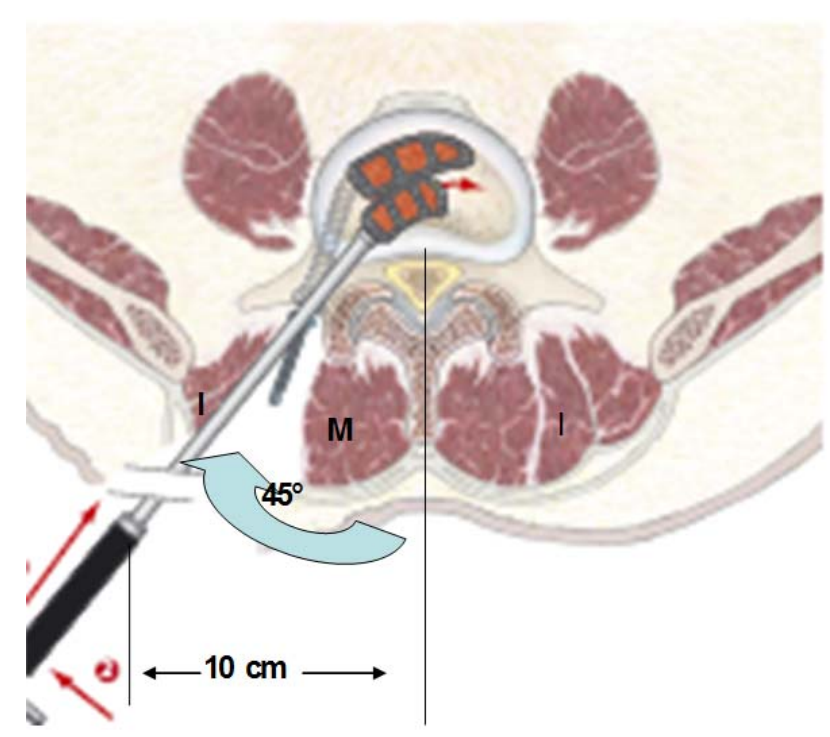




\section{Conclusion}

The muscle patterns, genetically controlled, is specific to each level of the lumbar spine. The figures of the three muscles (M, L and IC) surfaces, measured with CT horizontal cross sections, match one vertebral level. That guides the clinician to study the lumbosacral malformation and the surgeon to undergo extraforaminal intermuscular discal surgery. The ELIF makes it possible to insert two special shaped cages in the disc with a path between the muscles. This explanation shows that this technique respects the muscles and can be considered as a really mini invasive technique.

\section{Competing interests}

The authors declare that they have no competing interests.

\section{References}

[1] Recoules-Arche D, Alcaix D. Fusion lombaire intersomatique par voie extraforaminale unilatérale (ELIF unilatérale). A propos de 75 cas. Rachis 2, 4. 2006.

[2] Recoules-Arche D, Alcaix D. Extraforaminal Lumbar Interbody Fusion (ELIF) 30 degenerative lumbar dislocations. 1 year follow-up. Rachis 16. 2004.

[3] Mahato NK. Morphometric analysis and identification of characteristic features in sacra bearing accessory articulations with L5 vertebrae. 2010; Spine J 10: 616-621. PMid: 20620982. http://dx.doi.org/10.1016/j.spinee.2010.05.003

[4] Konin GP, Walz DM. Lumbosacral transitional vertebrae: classification, imaging findings, and clinical relevance. Am J Neuroradiol 15. 2010.

[5] Hughes RJ, Saifuddin A. Imaging of lumbosacral transitional vertebrae. Clin Radiol. 2004; 59: 984-991. PMid: 15488846. http://dx.doi.org/10.1016/j.crad.2004.02.019

[6] Bogduk N. Clinical anatomy of the lumbar spine and sacrum. Churchill Livingstone. 1997.

[7] Fortin M., Battie M.C. Quantitative paraspinal measurements. Phys. Ther. 1992; 6: 853-64.

[8] Hoh DJ, Wang MY, Rithland SL. Anatomic features of the paramedian muscle - splitting approach to the lumbar spine. Neurosurgery. 2010; 66: 13-24. PMid: 20124927. http://dx.doi.org/10.1227/01.NEU.0000350866.25760.33

[9] Zákány J, Gérard M, Favier B, et al. Deletion of a HoxD enhancer induces transcriptional heterochrony leading to transposition of the sacrum. EMBO J. 1997; 16: 4393-4402. PMid: 9250683. http://dx.doi.org/10.1093/emboj/16.14.4393

[10] Soshmikova N, Duboule D. Epigenetic control of hox genes collinear activation during vertebrate development. Mecanisms of Development. 209; 96-97.

[11] Abitbol MM. Evolution of the sacrum in hominoids. Am J Phys Anthropol. 1987; 74: 65-81. PMid: 3688211. http://dx.doi.org/10.1002/ajpa.1330740107 